\title{
Prediction of the Exit Site of Ventricular Tachycardia Based on Different ECG Lead Systems
}

\author{
Michał Kania ${ }^{1,4,6}$, Yves Coudière ${ }^{1,4,6}$, Hubert Cochet $^{2,3,5,6}$, Michel Haïssaguerre ${ }^{2,3,5,6}$, Pierre Jaïs ${ }^{2,3,5,6}$, \\ Mark Potse ${ }^{1,4,6}$ \\ ${ }^{1}$ CARMEN, Inria Bordeaux Sud-Ouest, Talence, France \\ ${ }^{2}$ INSERM, CRCTB, U1045, Bordeaux, France \\ ${ }^{3}$ Univ. Bordeaux, CRCTB, U1045, Bordeaux, France \\ ${ }^{4}$ Université de Bordeaux, IMB, UMR 5251, Talence, France \\ ${ }^{5}$ Bordeaux University Hospital (CHU), Electrophysiology and Ablation Unit, Pessac, France \\ ${ }^{6}$ IHU LIRYC, Electrophysiology and Heart Modeling Institute, Foundation Bordeaux University, \\ Pessac-Bordeaux, France
}

\begin{abstract}
The effectiveness of a computer-based method for localization of arrhythmia exit sites was studied.

The proposed algorithm works on any set of 3 or more ECG leads. The QRS complex integral of an ectopic beat is reduced to principal components treated as coordinates of the exit site in ECG space and then projected to real space by a linear transformation. The accuracy of the method was tested on 5 patient-tailored models of human heart and torso. For each model $\sim 500$ simulations were run, each for different stimulus location. All locations were then estimated from simulated surface ECGs and method accuracy was investigated.

The algorithm performed better for the left ventricle than for the right ventricle. The group mean absolute and relative (to a neighboring stimulation site) localization errors in millimeters were: $11.5(S D=2.5), 2.6(S D=0.5)$ for the 252-lead ECG; 12.2 (SD=2.7), $2.7(S D=0.5)$ for the 12-lead ECG; and $11.7(S D=2.4), 2.7(S D=0.5)$ for the Frank VCG.

This study suggest that the proposed method can predict exit sites with a precision in the order of a centimeter. Low values of error for neighbouring activation sites suggest opportunity for algorithm improvement. The use of vectorcardiographic leads is enough to obtain a precision comparable to a 252-lead ECG.
\end{abstract}

\section{Introduction}

Catheter ablation is currently a second or third-line treatment for ventricular tachycardia. It can deliver a permanent cure, but the semi-invasive procedure requires considerable human effort and is technically challenging. The procedure could be sped up and facilitated with a tool that would indicate the position of the "exit site" of the tachycardia with respect to the actual position of the mapping catheter. Current electrocardiographic imaging (ECGI) and other inverse cardiac models have more ambitious goals and are too cumbersome to follow the pace of a clinical procedure. Moreover they rely on recordings with hundreds of electrodes, which is undesirable in a catheterization laboratory, as well as on accurate medical imaging. Therefore we developed a relatively simple method with few requirements and a modest purpose, namely to predict the location from which the ventricular activation appears to spread, in relation to the location of the tip of the mapping catheter. The method does not require any knowledge of the anatomy and can work with 3 or more ECG leads.

One such system was previously proposed by Potse et al. [1, 2]. That system was only tested for 64-channel ECG measurements, and had a two-dimensional solution space limited to the left-ventricular endocardium, while arrhythmia often arise from the right ventricle or from more epicardial locations. Moreover, their validation data were less accurate and less extensive than what can be obtained today.

The purpose of this paper is to perform an in-silico investigation of this method. The questions we try to answer here are 1) is a fully three-dimensional prediction possible within the ventricles; 2) what accuracy can be expected in different regions of the heart; and 3) is it possible to obtain satisfactory accuracy with a low number of ECG leads? The answers to these questions 
can guide the design of subsequent clinical evaluations.

\section{Materials and methods}

\subsection{Preparation of testing data}

To prepare a data set for testing of our method we used a realistic membrane-based reaction-diffusion model of a human heart and torso and realistic patient-tailored geometries.

The model geometries were based on high-resolution cardiac computed tomography (CT) images. The cardiac wall and its cavities, the lungs, and the torso surface were segmented from the CT data. From the segmentations surface descriptions of these organs were made (Fig. 1). Based on these surface descriptions a hexahedral mesh of the heart at $200 \mu \mathrm{m}$ resolution and a torso mesh with $1 \mathrm{~mm}$ resolution were created. From the same surface descriptions of the heart a reduced surface of the ventricular myocardium with $\sim 500$ nodes was created using the MMG software [3]. For each node a simulation of a single beat was performed, in which the model was activated using a stimulation current pulse at the node location.

Simulations of cardiac activity were performed using a monodomain reaction-diffusion model

$$
C_{\mathrm{m}} \frac{\partial V_{\mathrm{m}}}{\partial t}=\beta^{-1} \nabla \cdot\left(G \nabla V_{\mathrm{m}}\right)-I_{\text {ion }}
$$

where $\beta$ is the membrane surface to volume ratio, $C_{\mathrm{m}}$ is the membrane capacitance per unit area, $G$ is the monodomain conductivity tensor, and $I_{\text {ion }}$ is the sum of all transmembrane ionic currents, computed with the model for the human ventricular myocyte proposed by Ten Tusscher et al. [4]. The monodomain conductivity tensor $G$ was chosen as $G=G_{\text {in }} G_{\text {ex }} /\left(G_{\text {in }}+G_{\text {ex }}\right)$ with $G_{\text {in }}$ and $G_{\text {ex }}$ the intracellular and extracellular conductivity tensor fields, respectively, to mimic the conduction velocity that a bidomain model would obtain [5]. The fiber orientations used to compute the conductivity tensors were assigned with a rule-based method $[5,6]$. As in previous studies the computed transmembrane currents were integrated over cubes of $1 \mathrm{~mm}$ size in order to be inserted in the torso model at $1 \mathrm{~mm}$ resolution [7]. In this model the extracellular potential $\phi_{\mathrm{e}}$ was solved using the inserted current $I_{\mathrm{m}}$ as a boundary condition:

$$
\nabla \cdot\left[\left(G_{\mathrm{in}}+G_{\mathrm{ex}}\right) \nabla \phi_{e}\right]=I_{\mathrm{m}}
$$

The simulations were performed with the Propag-5 software $[5,8]$ and ran on 2000 CPU cores. Because of the large number of simulations $\phi_{\mathrm{e}}$ was only output for the model nodes corresponding to the 268 electrodes shown in figure 1 (252 electrodes of the CardioInsight acquisition system, 9 standard ECG electrodes and 7 Frank ECG electrodes).

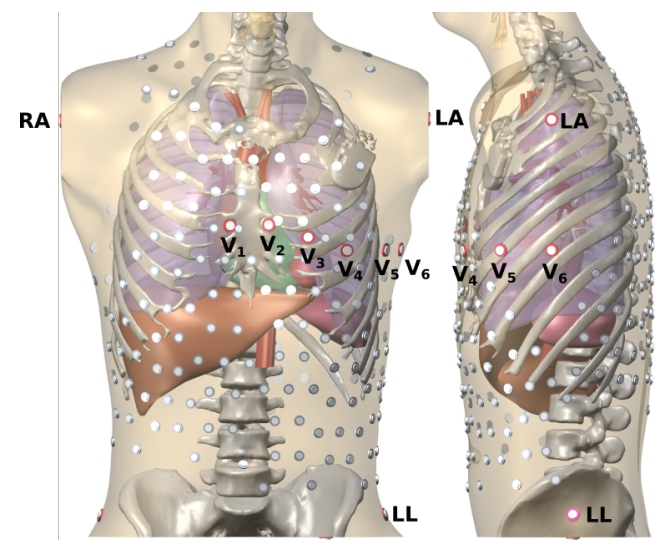

Figure 1. Segmented torso with internal organs and ECG electrode positions for one of studied subjects.

\subsection{Localization method}

The proposed localization method can work for any set of 3 or more ECG leads. It is based on the assumption that there is a linear relation between exit-site location and QRS-integral pattern on the thorax surface. The existence of a (not necessarily linear) relationship has been shown previously e.g. by SippensGroenewegen et al. [9]. Signals are integrated over the QRS interval of the ECG, to obtain a QRS integral vector. The first 3 principal components are taken and treated as coordinates of the exit site in ECG space. If there are just 3 leads, the SVD step is omitted.

Assuming that we know the exit site coordinates (either from the model or in reality using a paced beat from a position determined with an electroanatomical mapping system), we thus have a point $X$ in real space, and a corresponding point $E$ in ECG space. Using a limited number of paced beats (at least 3 ), we estimate the $3 \times 3$ transformation matrix $R$ and the 3 -element translation vector $T$ such that

$$
X \approx R E+T
$$

for each pair $(X, E)$. This problem is solved in a leastsquares sense. In a real application, this transform would be fitted to a small number of paced beats with known pacing locations. In this study, we fitted to all points and evaluated the fitting error, for different lead systems.

\subsection{Evaluation of accuracy}

We evaluated the accuracy of the method using 5 patient-tailored models. The accuracy of localization was quantified by computation of 1) the absolute errors $A b s E r r=d_{P P},(i)$, which are the distances between the $i^{\text {th }}$ stimulation site location $P(i)$ and its position estimated by our method $P^{\prime}(i)$; and 2$)$ the errors of relative localization RelErr $=\left|d_{P^{\prime} Q^{\prime}}(i)-d_{P Q}(i)\right|$, which quantify the error for 
localization with respect to a nearby location. Mesh nodes were considered neighboring when they had an edge in common. The relative error indicates how the method would perform if the catheter tip is already near the arrhythmia exit site and the cardiologist would like to move to the next estimated position for the exit site.

We investigated the influence of different ECG lead sets (252-lead ECG, standard 12-lead ECG and Frank vectorcardiographic (VCG) leads) on accuracy.

\section{Results}

\subsection{Representation of the ventricles in ECG space}

For all lead systems, the reduced ventricular model (Fig. 2,A) plotted in ECG space (Fig. 2,B) (using the original topology but replacing each set of $X$ coordinates by the corresponding $E$ ) could be recognized as a pair of ventricles.
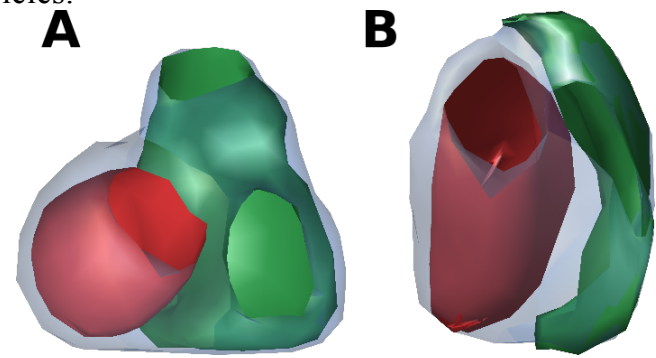

Figure 2. A - simplified heart mesh plotted from stimulation points positions $X$. B - heart geometry in ECG space plotted from estimated stimulation sites $E$ using a 252-lead ECG.

In all simulations, the shape of the left ventricle (LV) was well preserved, except for the papillary muscles which were more or less turned inside-out and protruded slightly outside the heart. The right ventricle (RV) was flattened, but the lateral wall was still separated from the septum. Endocardium and epicardium of the RV intersected, and the dimensions of the RV outflow tract (RVOT) were strongly reduced. Importantly, the interventricular septum, including the common outflow tract, appeared relatively thick, suggesting a good differentiation of LV from RV sites.

\subsection{Performance of different leads sets}

Fig. 3 shows the dependence of stimulation site location on localization errors for one of the models.

The group mean absolute and relative localization errors (in $\mathrm{mm}$ ) computed from all patient-specific models were: $11.5(\mathrm{SD}=2.5), 2.6(\mathrm{SD}=0.5)$ for the 252-lead $\mathrm{ECG}$; $12.2(\mathrm{SD}=2.7), 2.7(\mathrm{SD}=0.5)$ for the 12-lead $\mathrm{ECG}$; and $11.7(\mathrm{SD}=2.4), 2.7(\mathrm{SD}=0.5)$ for the Frank VCG.

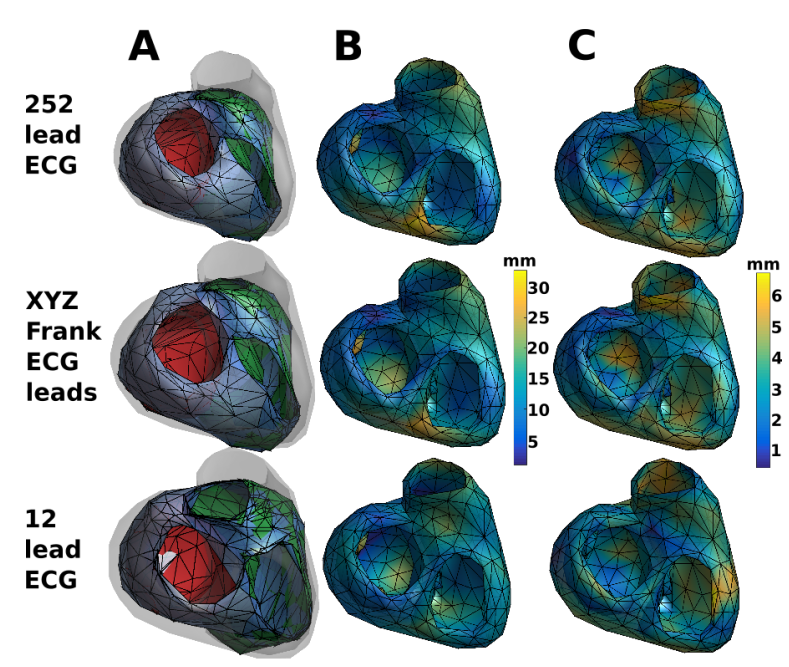

Figure 3. The accuracy of localization for one of the subjects. A - estimated positions of stimulation points in real space; B - absolute localization errors; C - errors of relative localization.

The use of a Frank VCG led to comparable algorithm performance as in case of full 252-lead ECG. The mean values of localization errors in different regions in the heart in case of Frank XYZ leads are summarized in Fig. 4. The trends of error values depending on stimulation site location for other ECG lead systems were similar.
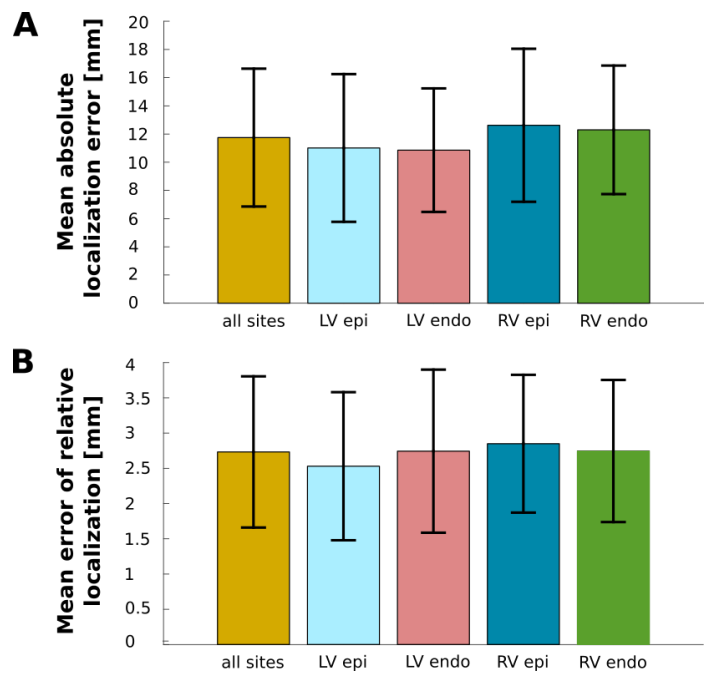

Figure 4. Mean absolute localization errors (A) and mean errors of relative localization (B) for all patients with Frank VCG used as an input in localization procedure.

The mean errors for the right ventricle were higher than for the left ventricle. This can also be seen in Fig. 3A where for one of the patients the estimated stimulation sites are plotted together with the original mesh made 
from known stimulation points (grey, transparent mesh). The fitting is satisfactory for the left ventricle while the right ventricle is more deformed. In all cases the distribution of the error values was smooth (Fig. 3, panels $\mathrm{B}$ and $\mathrm{C})$.

\section{Discussion}

A computer algorithm based on a linear relation between surface ECGs and exit site can predict exit sites with a precision in the order of a centimeter for absolute errors and in order of three millimeters for errors of relative localization. The use of an XYZ ECG-lead system is enough to get satisfactory results, with a precision of localization comparable to full 252-lead ECG. This makes it easier to implement the method in clinical settings. Low values of errors of relative localization and its smooth distribution on the heart surface suggest opportunity for algorithm improvement. The obtained results will guide the design of subsequent clinical evaluations.

A limitation of the current study is that we always reduced the QRS integral to 3 principal components. In principle the method could also use a larger number. However, initial tests showed that the problem to solve the transform $(R, T)$ quickly became ill-conditioned with higher-dimensional inputs $E$. A further limitation is that we did not assess the influence of factors such as ECG noise and the use of small sets of fitting positions. These are topics for future work.

\section{Acknowledgements}

The research leading to these results has received funding from the European Research Council under the European Union's Seventh Framework Programme (FP/2007-2013) / ERC Grant Agreement nr. ERC-2012ADG 20120314.

This work was granted access to HPC resources of CINES and CEA-TGCC under GENCI allocation x2016037379.

\section{References}

[1] Potse M, Linnenbank AC, Peeters HA, SippensGroenewegen A, Grimbergen CA. Continuous localization of cardiac activation sites using a database of multichannel ECG recordings. IEEE Trans Biomed Eng. 2000;47:682-9.

[2] Potse M, Hoekema R, Linnenbank AC, SippensGroenewegen A, Strackee J, de Bakker JMT, et al. Conversion of left ventricular endocardial positions from patient-independent co-ordinates into biplane fluoroscopic projections. Med Biol Eng Comput. 2002;40:41-6.

[3] Dapogny C, Dobrzynski C, Frey P. Three-dimensional adaptive domain remeshing, implicit domain meshing, and applications to free and moving boundary problems. Journal of computational physics. 2014;262:358-78.

[4] ten Tusscher KHWH, Noble D, Noble PJ, Panfilov AV. A model for human ventricular tissue. Am J Physiol Heart Circ Physiol. 2004;286:H1573-89.

[5] Potse M, Dubé B, Richer J, Vinet A, Gulrajani RM. A comparison of monodomain and bidomain reactiondiffusion models for action potential propagation in the human heart. IEEE Trans Biomed Eng. 2006;53:2425-35.

[6] Streeter DD, Jr., Spotnitz HM, Patel DP, Ross J, Jr., Sonnenblick EH. Fiber orientation in the canine left ventricle during diastole and systole. Circ Res. 1969;24:339-47.

[7] Potse M, Dubé B, Vinet A. Cardiac anisotropy in boundary-element models for the electrocardiogram. Med Biol Eng Comput. 2009;47:719-29.

[8] Krause D, Potse M, Dickopf T, Krause R, Auricchio A, Prinzen F. Hybrid parallelization of a large-scale heart model. Facing the Multicore-Challenge II: Springer; 2012. p. 120-32.

[9] SippensGroenewegen A, Spekhorst H, van Hemel NM, Kingma JH, Hauer RN, Janse MJ, et al. Body surface mapping of ectopic left and right ventricular activation. QRS spectrum in patients without structural heart disease. Circulation. 1990;82:879-96.

Address for correspondence.

Michal Kania

Electrophysiology and heart modeling institute LIRYC

F-33600 Pessac-Bordeaux, France

michal.kania@ihu-liryc.fr 